\title{
La estructura del perifiton y de la deriva algal en arroyos serranos.
}

\author{
Noemí Gari1 y María del C. Corigliano² \\ Departamento de Ciencias Naturales, Universidad Nacional de Río Cuarto. Ruta Nacional 36, Km. 601. \\ Campus Universitario. 5800- Río Cuarto. República Argentina. E-mail: ngari@exa.unrc.edu.ar ${ }^{1}$ mcori- \\ gliano@exa.unrc.edu.ar ${ }^{2}$
}

\section{RESUMEN}

Se analiza la relación estructural entre perifiton y deriva algal, en la confluencia de arroyos serranos de diferente orden. Se realizaron muestreos mensuales durante un ciclo anual en cuatro sitios ubicados en el área de confluencia de los arroyos Piedra Blanca y San Bartolomé, de la cuenca alta del Río Chocancharava, Córdoba, Argentina. Se identificaron 166 taxa, entre especies, variedades y formas. La mayor riqueza específica correspondió a la clase Bacillariophyceae. La densidad máxima de deriva fue de $40.58 \mathrm{org} . / \mathrm{ml}$. Tratamientos con ANOVA y test de Kruskal-Wallis evidenciaron las diferencias espaciotemporales en densidad, riqueza y diversidad de ambas comunidades. La diversidad y riqueza de especies fueron mayores en la deriva. La clasificación de las muestras por TWINSPAN separó a los dos ensambles en grupos diferentes, las muestras se ordenaron siguiendo un gradiente de temperatura y velocidad de corriente, mientras que las especies lo hicieron, según su predominio en cada comunidad. El perifiton del arroyo de mayor orden influyó sobre la composición y atributos estructurales de la deriva del río receptor. En la densidad de deriva predominan las poblaciones procedentes del perifiton mientras que en la composición, la alta riqueza específica es de origen múltiple y heterogéneo.

Palabras clave: perifiton, deriva, arroyos, algas

\begin{abstract}
The structural relationships between periphyton and algae drift are analyzed in the confluence of two mountain streams of different order. Samples were taken monthly, over a one-year cycle in four sites at the confluence of Piedra Blanca and San Bartolome streams in the higher Chocancharava river basin, Cordoba, Argentina. A total of 166 taxa were identified (species, varieties and morfo-species). The highest specific richness corresponded to Bacillariophyceae. Maximum drift density was $40.58 \mathrm{org} . \mathrm{ml}$. ANOVA and test of Kruskal-Wallis treatments showed the spatial and temporal differences in density, species richness, and diversity of both communities. Diversity and species richness were highest in drift. TWINSPAN classification of samples separated the assemblages in two different sets. Samples' ordination happened along thermal and current velocity gradients, while species ordination was according to the community where they predominated. The periphyton from the highest order stream influenced the composition and structural attributes of drift in the receiving stream. Periphyton populations predominate in drift's density, whereas in drift species composition, richness is of a multiple and heterogeneous origin.
\end{abstract}

Key words: periphyton, drift, streams, algae

\section{INTRODUCCIÓN}

En pequeñas corrientes fuertes, el desplazamiento de autótrofos del perifiton determina que, en la columna de agua, las algas se encuentren en transporte mezcladas con el ensamble fitoplanctónico originado en las aguas tranquilas. La composición de las algas epilíticas varía según el tamaño del sustrato ya que su manera de adherirse y forma de crecimiento condicio- nan su distribución y desplazamiento. Existen algas libres en el sedimento, con mayor o menor movilidad según las horas del día y son las que, periódicamente, dando origen a pulsos regulares de deriva, pueden formar parte del potamoplancton (Allan, 1995).

La emigración-inmigración y posterior colonización, son procesos importantes que regulan la composición de diatomeas perifíticas y la productividad primaria de los arroyos e influyen 
sobre los atributos de las comunidades algales y en la red trófica fluvial (Merrit \& Wallace, 1981; Allan, 1995). Además, la densidad de las algas que se acumulan diariamente sobre el sustrato, especialmente durante los estados tempranos y tardíos de desarrollo de la comunidad, son el resultado de la inmigración (Stevenson, 1984; 1986; 1990; Stevenson \& Peterson, 1989). Algunas de las variaciones interespecíficas en las tasas de inmigración temprana, pueden ser explicadas por variaciones interespecíficas en la abundancia del transporte algal (MüllerHaeckel, 1976; Stevenson, 1983).

En general, los trabajos sobre el componente algal en transporte, en arroyos, no se han dedicado a relacionar cuánto de éste es plancton verdadero y cuánto corresponde a organismos del perifiton, arrastrados por la corriente río abajo. Los estudios más detallados al respecto han dado cuenta que el perifiton contribuye con una participación importante en la abundancia de deriva. (Müller-Haeckle, 1976; 1978 )

En los ríos serranos de la Sierra de Comechingones, cuenca tributaria superior de los ríos Chocancharava y Ctalamochita, provincia de Córdoba, las algas epilíticas y el componente en transporte presentan una alta riqueza de especies, en particular de diatomeas (Luque \& Martínez de Fabricius, 2000). Se ha observado que el componente en transporte está constituido por efectivos planctónicos, procedentes desde remansos, hoyas o embalses, pero, una gran fracción del número de especies son formas derivantes del perifiton (Martínez de Fabricius et al., 1988; Martínez de Fabricius \& Corigliano, 1989) siendo la más frecuentes y abundantes Navicula, Nitzschia y Cymbella (Corigliano et al., 1994).

En una red hídrica los afluentes tributarios son modificadores del continuo fluvial (Bruns et al.,1984), ya que originan cambios hidráulicos (Statzner, \&. Higler, 1986) y encuentro de distintos conjuntos de especies en las confluencias (Margalef, 1994). El análisis de la relación perifiton deriva en una zona de confluencia, permite desarrollar observaciones con respecto a la continuidad o discontinuidad desde aguas arriba y conocer, cómo la composición del perifiton río abajo es afectada por la deriva, vía los procesos de migración y colonización. El objetivo de este trabajo es analizar la relación de la composición y estructura del perifiton y de la deriva algal en la confluencia de arroyos serranos de diferente orden, y su efecto sobre el río colector.

\section{ÁREA DE ESTUdIO}

El área de estudio se encuentra ubicada en la cuenca superior del río Chocancharava en la provincia de Córdoba, Argentina. Esta cuenca drena una superficie aproximada de 200000 ha, desde la Sierra de Comechingones hasta los Bajos del Saladillo. Se la subdivide en cuenca alta en el área serrana, cuenca media en la llanura aluvial ancha y cuenca baja en el área de derrame. Los sitios de estudio están localizados en la transición entre cuenca alta y media, zona de piedemonte. La primera se desarrolla sobre rocas de basamento cristalino de edad precámbrica a paleozoica inferior. La litología es de baja a nula permeabilidad y el relieve de pendientes pronunciadas, la red de drenaje es de alta densidad con alta frecuencia de cursos. Estas características sumadas a la intensidad de las precipitaciones definen escurrimientos superficiales con rápido tiempo de concentración e importantes picos de crecidas. Son cursos de régimen torrencial con precipitación anual media de $901 \mathrm{~mm}$ (Vázquez et al., 1979), localizados en el Dominio Chaqueño, predominantemente bosque serrano degradado por acción del fuego, tala y pastoreo. En la actualidad existen fases de reemplazo integradas especialmente por especies exóticas en las posiciones ripariales.

\section{MATERIAL Y MÉTODOS}

Los sitios de muestreo se encuentran en los tramos de confluencia de los arroyos Piedra Blanca y San Bartolomé. (Tabla 1). El sitio 1 (PB) está ubicado en el arroyo Piedra Blanca a $100 \mathrm{~m}$ aguas arriba de la confluencia, el sitio 2 (SB) en el arroyo San Bartolomé a 200 m aguas 


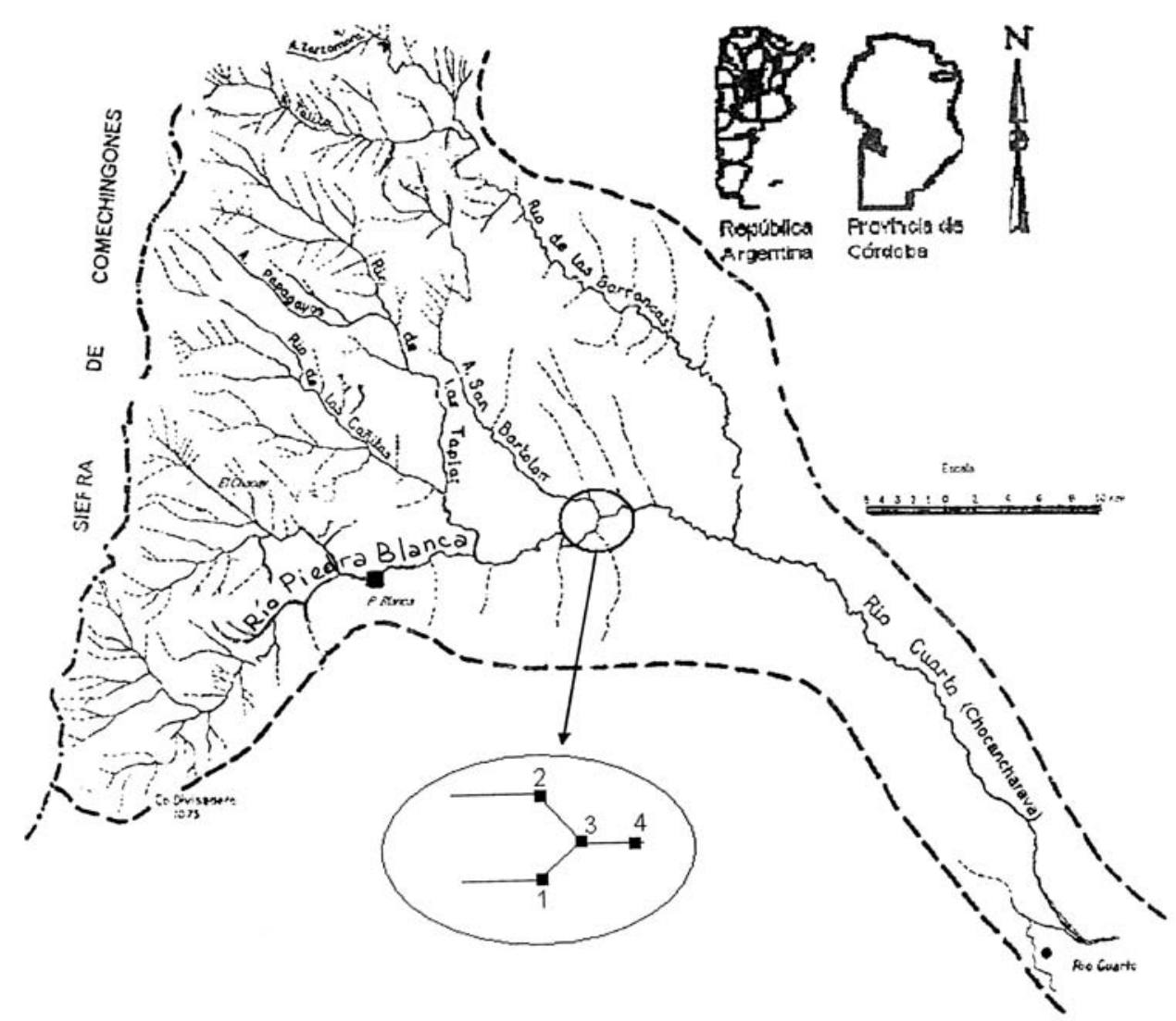

Figura 1. Sitios de muestreo en la confluencia de los arroyos Piedra Blanca y San Bartolomé, cuenca del río Chocancharava, Córdoba, Argentina. Sampling sites at the confluence of Piedra Blanca and San Bartolomé streams, Chocancharava basin, Cordoba, Argentina.

arriba de la confluencia, el sitio $3(\mathrm{CO})$ en la confluencia de los arroyos y el sitio $4(\mathrm{CH})$ en el río Chocancharava a $200 \mathrm{~m}$ aguas abajo de la confluencia (Fig. 1).

En cada uno de los sitios se registraron variables hidráulicas: velocidad de corriente, con correntímetro digital Global Flow Probe, ancho y profundidad del cauce; variables físico-químicas: temperatura del agua, del aire, $\mathrm{pH}$ con sensor $\mathrm{P}$. Altronix M-206, conductividad y sólidos disueltos con sensor Altronix CT2. Se determinó la granulometría del sedimento según Hynes (1970) y el número de orden de los arroyos según Strahler (1981). Las determinaciones químicas de las aguas se realizaron semestralmente, en aguas bajas y altas, siguiendo las técnicas de APHA (1989).

Las muestras de deriva se tomaron mensualmente, en el centro del canal de cada sitio, desde abril de 1994 a mayo 1995 con red de plancton, de $25 \mu \mathrm{m}$ de apertura de malla. Las muestras cualitativas se obtuvieron por método de arrastre manual y las cuantitativas filtrando de 100 a 200 litros de agua a través de red, teniendo en cuenta el mismo rango horario. Las muestras de perifiton se recolectaron en una corredera de los sitios de muestreo 1, 2 y 4, por el método estratificado al azar, mediante el raspado de una superficie de $100 \mathrm{~cm}^{2}$, (Whitton, 1991). En el sitio 3 no se obtuvieron muestras de perifiton por no ser accesible el sustrato durante aguas altas.

Para el análisis taxonómico se utilizó bibliografía específica de cada grupo y/o sección en particular: Desikachary, (1959); Prescott, (1962); Patrick \& Reimer, (1966, 1975); Germain, (1981); Archibald, (1983); Tell \& 
Tabla 1. Parámetros físicos y químicos, en los sitios de muestreo de la subcuenca del río Chocancharava (Córdoba, Argentina). 1: arroyo Piedra Blanca; 2: arroyo San Bartolomé; 3: Confluencia, 4: río Chocancharava. Valores promedios y rangos, n=13. Physical and Chemical parameters in the study sites in the sub-Chocancharava basin (Cordoba, Argentina). 1: Piedra Blanca stream, 2: San Bartolome stream, 3: Confluence 4: Chocancharava river. Average values and ranges, $n=13$.

\begin{tabular}{|c|c|c|c|c|}
\hline \multirow{2}{*}{ CARACTERISTICAS } & \multicolumn{4}{|c|}{ Sitios de Muestreo } \\
\hline & 1 & 2 & 3 & 4 \\
\hline Ubicación & \multicolumn{4}{|c|}{$32^{\circ} 56^{\prime} \mathrm{S}$ y $64^{\circ} 41^{\prime} \mathrm{O}$} \\
\hline Altitud & \multicolumn{4}{|c|}{550} \\
\hline Profundidad (m) & $\begin{array}{c}0.47 \\
(0.15-0.70)\end{array}$ & $\begin{array}{c}035 \\
(0.1-0.4)\end{array}$ & $\begin{array}{c}0.55 \\
(0.3-0.8)\end{array}$ & $\begin{array}{c}0.6 \\
(0.2-1)\end{array}$ \\
\hline Veloc. Corriente (m.seg $\left.{ }^{-1}\right)$ & $\begin{array}{c}0.67 \\
(0.5-1)\end{array}$ & $\begin{array}{c}0.67 \\
(0.4-0.8)\end{array}$ & $\begin{array}{c}0.66 \\
(0.33-1)\end{array}$ & $\begin{array}{c}0.79 \\
(0.33-1.33)\end{array}$ \\
\hline Cauce Húmedo (m) & 15 & 10 & 15 & 15 \\
\hline Caudal Medio anual $\left(\mathrm{m}^{3} \cdot \mathrm{seg}^{-1}\right)(1)$ & 3.52 & 1.09 & 3.7 & 3.7 \\
\hline Gradiente del río & 0.19 & 0.16 & 0.16 & 0.16 \\
\hline Superficie Cuenca $\left(\mathrm{Km}^{2}\right)^{(1)}$ & 340 & 270 & & 1450 \\
\hline Longitud del río (Km) & 130 & 90 & & \\
\hline $\mathrm{N}^{\mathrm{o}}$ de Orden ${ }^{(3)}$ & 6 & 3 & 6 & 6 \\
\hline $\mathrm{N}^{\mathrm{o}}$ de Ligamientos & 139 & 18 & 157 & 157 \\
\hline Sedimento dominate ${ }^{(2)}$ & $\begin{array}{c}\text { Bloque } \\
\text { (Guijaro-Grava) }\end{array}$ & $\begin{array}{c}\text { Guijarro } \\
\text { (Grava-arena) }\end{array}$ & $\begin{array}{c}\text { Bloque } \\
\text { (Guijarro-grava) }\end{array}$ & $\begin{array}{c}\text { Bloque } \\
\text { (Guijarro-grava) }\end{array}$ \\
\hline Temperatura Agua $\left({ }^{\circ} \mathrm{C}\right)$ & $\begin{array}{l}15.15 \\
(9-22)\end{array}$ & $\begin{array}{c}18.69 \\
(11-25)\end{array}$ & $\begin{array}{c}17.31 \\
(10-25)\end{array}$ & $\begin{array}{c}15.62 \\
(10-24)\end{array}$ \\
\hline Conductividad $\left(\mu \mathrm{Scm}^{-1)}\right)^{(1)}$ & $\begin{array}{c}174 \\
(70-339)\end{array}$ & $\begin{array}{c}129.42 \\
(109-133)\end{array}$ & $\begin{array}{c}147.9 \\
(64-203)\end{array}$ & $\begin{array}{c}154.7 \\
(65-202)\end{array}$ \\
\hline $\mathrm{pH}$ & $\begin{array}{c}7.49 \\
(6.5-8.5)\end{array}$ & $\begin{array}{c}7.63 \\
(6.5-8.7)\end{array}$ & $\begin{array}{c}7.56 \\
(7-8.5)\end{array}$ & $\begin{array}{c}7.5 \\
(6.5-8.5)\end{array}$ \\
\hline Sólidos Disueltos (ppm) & $\begin{array}{c}89.23 \\
(30-120)\end{array}$ & $\begin{array}{c}68.3 \\
(50-80)\end{array}$ & $\begin{array}{c}80 \\
(40-120)\end{array}$ & $\begin{array}{c}90 \\
(30-120)\end{array}$ \\
\hline $\begin{array}{l}\text { (1) Registros tomados de Agua y E } \\
\text { (2) Clasificación de sedimentos ton } \\
\text { (3) Clasificación según Strahler (1 }\end{array}$ & $\begin{array}{l}\text { a (1987) } \\
\text { de Hynes (1980) }\end{array}$ & & & \\
\hline
\end{tabular}

Conforti, (1986); Krammer \& Lange-Bertalot, (1986, 1988, 1991a, 1991b), Round, Crawford \& Mann (1990), Round \& Bukhtiyarova (1996), donde se incluyen sinonimias posteriores al tratado de Van Landingham, (1967-1979).

Los recuentos se realizaron por transectas según el método propuesto por Villafañe \& Reid (1995). Se predeterminaron tres transectas horizontales equidistantes evitando los efectos de bordes. Se utilizó portaobjetos y cubreobjeto de $24 \times 50 \mathrm{~mm}$, en el portaobjeto se sedimentó una alícuota de $0.3 \mathrm{ml}$, esto permitió la observación del material con 400 aumentos. El número de cámaras contadas, hasta no registrar nuevas especies, fue de 5 para cada muestra. Se consideró como unidad de recuento al organismo (colonia, unicelular, cenobio, filamento). Para los filamentos, el equivalente a organismo fue la longitud promedio de un organismo unicelular frecuente como Synedra y/o Fragilaria. Los cálculos de densidad se expresaron para deriva en org $/ \mathrm{ml}$ y en $\mathrm{org} / \mathrm{cm}^{2}$ para perifiton.

Se aplicaron los índices de: Similitud de Jaccard, diversidad H'de Shannon-Weaver, riqueza de especies, usando el programa Statistical Ecology (Ludwig \& Reynolds, 1988). La clasificación de las especies y muestras se realizó con TWINSPAN (Hill,1979a) y el orde- 
Tabla 2. Variables químicas en los sitios de estudio en aguas bajas (junio) y aguas altas (marzo).1: arroyo Piedra Blanca; 2: arroyo San Bartolomé; 3: Confluencia, 4: río Chocancharava. Chemical variables at study sites during low (June) and high (March) water levels. 1: Piedra Blanca stream, 2: San Bartolome stream, 3: Confluence, 4: Chocancharava river.

\begin{tabular}{cccccccccc}
\hline $\begin{array}{c}\text { SITIO } \\
\text { junio/97 }\end{array}$ & $\begin{array}{c}\mathrm{CO}_{3}= \\
\mathrm{mg} / 1\end{array}$ & $\begin{array}{c}\mathrm{CO}_{3} \mathrm{H}^{-} \\
\mathrm{mg} / \mathrm{l}\end{array}$ & $\begin{array}{c}\mathrm{SO}_{4}= \\
\mathrm{mg} / 1\end{array}$ & $\begin{array}{c}\mathrm{Cl}^{-} \\
\mathrm{mg} / 1\end{array}$ & $\begin{array}{c}\mathrm{Na}^{+} \\
\mathrm{mg} / 1\end{array}$ & $\begin{array}{c}\mathrm{K}^{+} \\
\mathrm{mg} / 1\end{array}$ & $\begin{array}{c}\mathrm{Ca}^{++} \\
\mathrm{mg} / 1\end{array}$ & $\begin{array}{c}\mathrm{Mg}^{++} \\
\mathrm{mg} / 1\end{array}$ & $\begin{array}{c}\mathrm{F}^{-} \\
\mathrm{mg} / 1\end{array}$ \\
\hline 1 & 0.00 & 88.50 & 16.91 & 7.71 & 11.16 & 3.08 & 21.60 & 5.61 & 0.30 \\
2 & 0.00 & 56.55 & 12.59 & 5.14 & 5.35 & 3.08 & 15.60 & 3.66 & 0.42 \\
3 & 0.00 & 87.50 & 12.59 & 5.14 & 10.31 & 2.93 & 20.00 & 6.34 & 0.30 \\
4 & 4.85 & 70.00 & 31.86 & 5.14 & 11.12 & 2.93 & 22.00 & 7.56 & 0.26 \\
\hline Marzo/98 & & & & & & & & & \\
1 & 0.00 & 75.00 & 20.85 & 5.71 & 12.10 & 2.64 & 16.00 & 7.32 & 0.45 \\
2 & 0.00 & 47.50 & 23.60 & 3.71 & 10.40 & 2.26 & 14.40 & 3.41 & 0.73 \\
3 & 0.00 & 82.50 & 25.17 & 5.71 & 12.29 & 1.95 & 19.20 & 5.85 & 0.43 \\
4 & 0.00 & 75.00 & 23.21 & 6.00 & 12.29 & 1.85 & 19.20 & 3.90 & 0.43 \\
\hline
\end{tabular}

namiento de las especies y muestras con las variables ambientales: temperatura y velocidad de corriente y las variables estructurales: densidad, diversidad y riqueza de especies, por Análisis de Correspondencia Detendenciado (DCA) con DECORANA (Hill, 1979b). Se incluyeron aquellas especies cuya abundancia relativa fue $\geq 1 \%$ en por lo menos una de las muestras. Los datos originales fueron estandarizados a porcentaje para permitir la comparación entre perifiton y deriva. Previa verificación de las condiciones de normalidad y homocedasticidad de los datos (Test de Kolmogorov Smirnov, test de Cochran y test de Barlett- Box), se utilizó el análisis de la varianza univariado (ANOVA), para comparar muestras espaciales y temporales. Los datos de abundancia del perifiton y deriva se transformaron a $\log (\mathrm{x}+1)$ para estabilizar la varianza. Para los datos de riqueza y diversidad del perifiton, que no se normalizaron con la transformación, se aplicó el test de Kruskal-Wallis (K-W).

\section{RESULTADOS}

Los sitios estudiados se caracterizaron por presentar valores de velocidad de corriente dentro de rangos y promedios que van de $0.33 \mathrm{~m} / \mathrm{s} \mathrm{a}$ $1 \mathrm{~m} / \mathrm{s}$, mientras que la profundidad aumentó desde los arroyos de menor orden, promedio
$0.10 \mathrm{~m}$ hasta $1 \mathrm{~m}$ en el río colector. Se observaron amplios rangos de variación en cada sitio a lo largo del año indicativo de las condiciones de estiaje y crecientes.

El pH, alcanzó valores circumneutrales a ligeramente alcalinos, con un máximo de 8.7 en los meses de setiembre y octubre. Los mayores valores de conductividad fueron de $339 \mu \mathrm{S} / \mathrm{cm}$ y los menores de $65 \mu \mathrm{S} / \mathrm{cm}$, los sólidos disueltos alcanzaron $120 \mathrm{ppm}$ (Tabla 1). Aplicando ANOVA o $\mathrm{K}-\mathrm{W}$ se registraron diferencias significativas $(\mathrm{p} \leq 0.01)$, entre los sitios de estudio en la conduc-

\begin{tabular}{rrrrrrrrr}
\hline & 1D & 2D & 3D & 4D & 1P & 2P & 4P \\
\hline 1D & 1 & & & & & & & \\
2D & 0,55 & 1 & & & & & & \\
3D & 0,50 & 0,44 & 1 & & & & & \\
4D & 0,47 & 0,46 & 0,52 & 1 & & & \\
1P & 0,32 & 0,31 & 0,40 & 0,40 & 1 & & \\
2P & 0,36 & 0,37 & 0,37 & 0,38 & 0,64 & 1 & \\
4P & 0,25 & 0,27 & 0,33 & 0,35 & 0,60 & 0,49 & 1 \\
\hline
\end{tabular}

\section{$\%$ de Similitud}

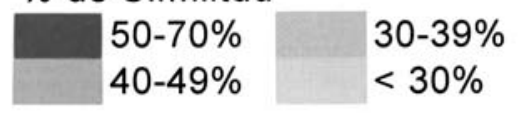

Figura 2. Matriz de Similitud de Jaccard de los sitios estudiados de la confluencia de los arroyos Piedra Blanca y San Bartolomé, 1994-95. Jaccard Similitary Matrix of the study sites at the confluence of Piedra Blanca and San Bartolome streams, 1994-95. 
Tabla 3. Lista de taxones algales en deriva y perifiton en la confluencia de los arroyos Piedra Blanca y San Bartolomé, en el período 1994-95. El asterisco * indica abundancia $>1 \%$ en las muestras y las abreviaturas se usan para las etiquetas de los análisis estadísticos. List of algae taxa in drift, and periphyton at the confluence of Piedra Blanca and San Bartolome streams, during 1994-95. The asterisk* designates abundance $>1 \%$ in the samples, and abbreviation are used for statistical analysis labels.

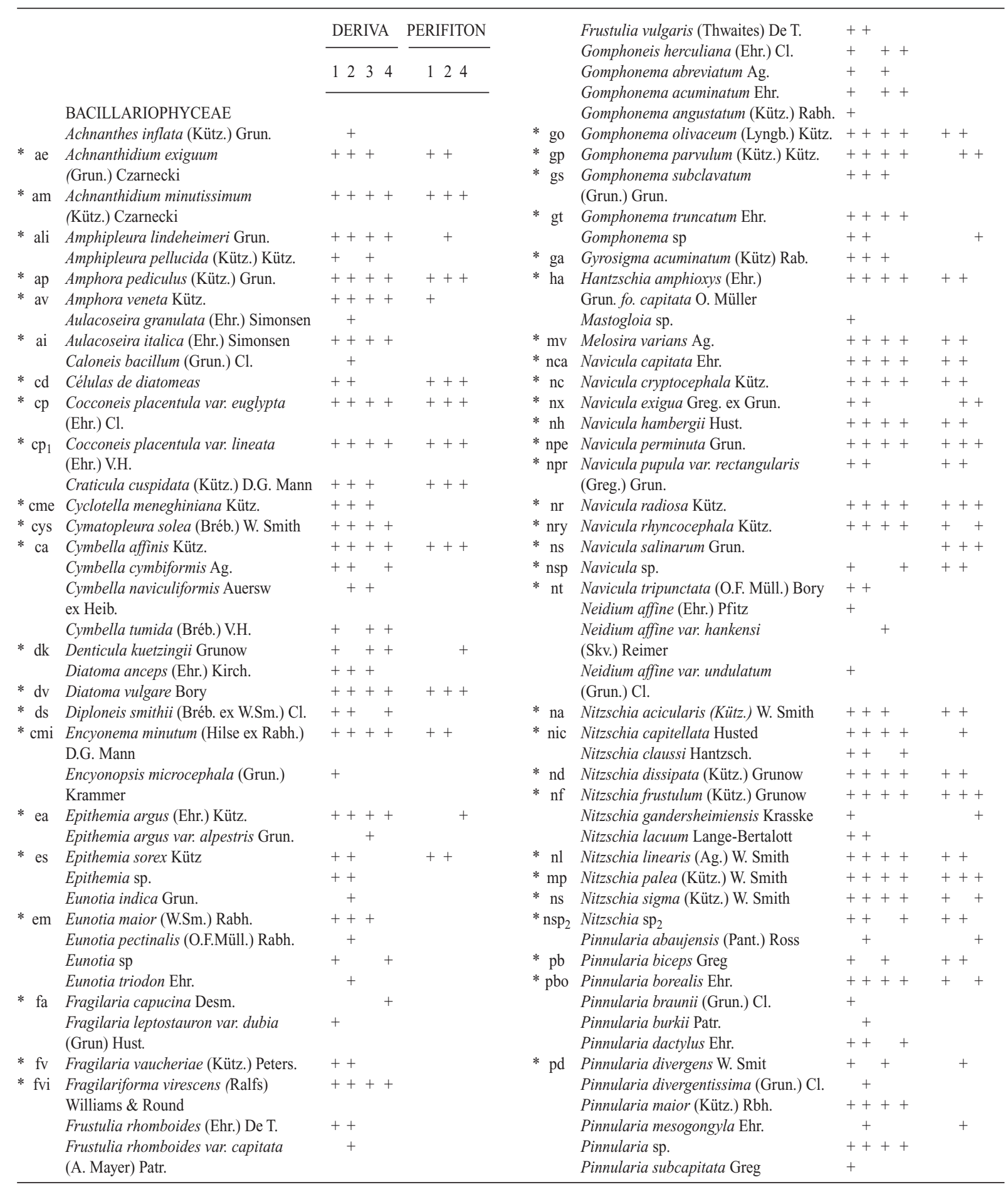




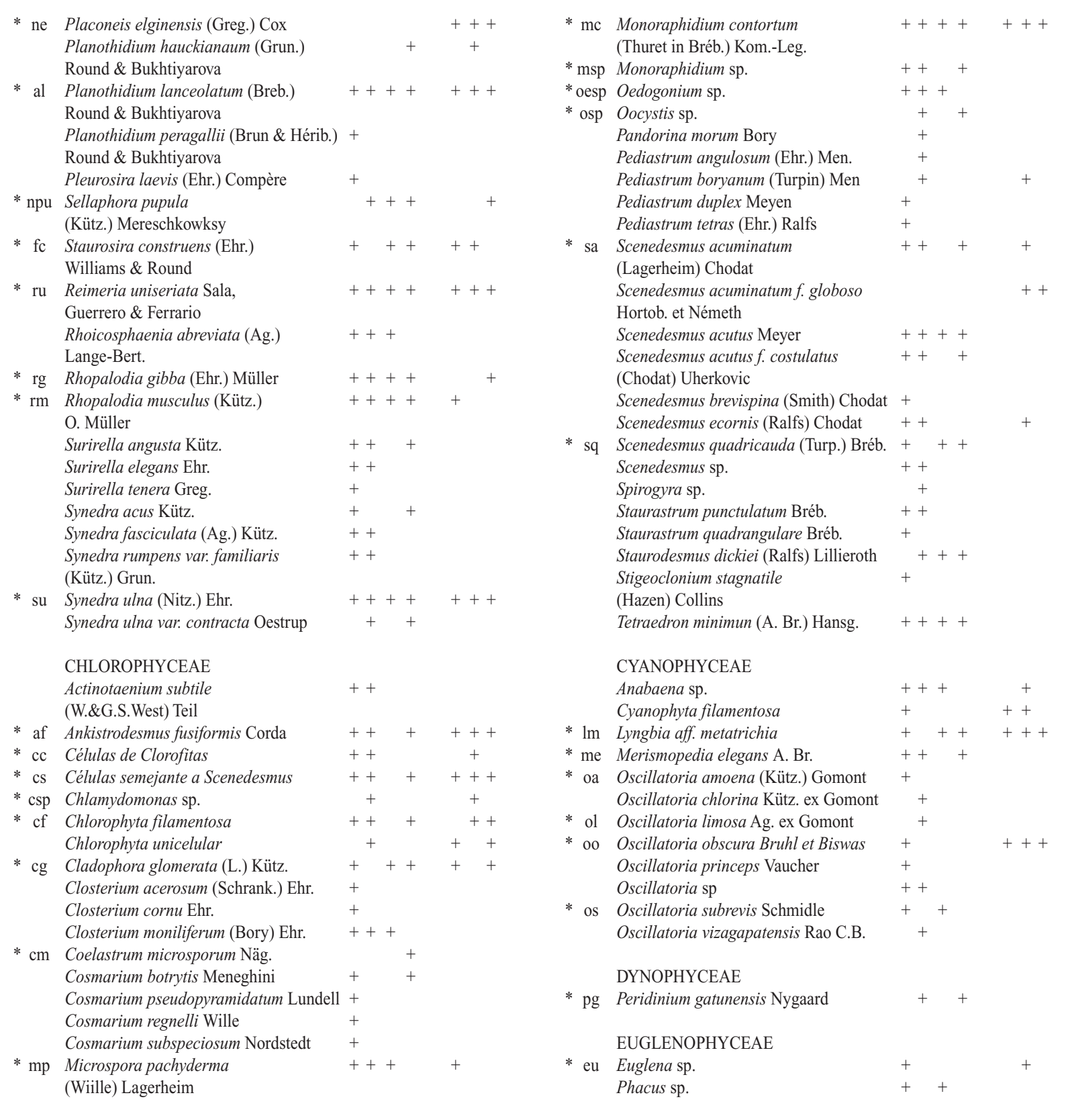

tividad y los sólidos disueltos y en las series de datos mensuales, en la velocidad de corriente, la temperatura, el pH, la conductividad y los sólidos disueltos. La carga iónica mostró diferencias entre los sitios 1 y 2 . Se categorizaron las aguas como bicarbonato-cálcicas, con: $\mathrm{CO}_{3} \mathrm{H}^{-}$ $>>\mathrm{SO}_{4}=>\mathrm{Cl}^{-}$y $\mathrm{Ca}^{++}>\mathrm{Na}^{+}>\mathrm{Mg}^{++}>\mathrm{K}^{+}$(Tabla 2 ).
Se identificaron un total de 166 especies (Tabla 3). La distribución de la riqueza específica por clase fue: Bacillariophyceae 111 especies; Chlorophyceae 40, Cyanophyceae 12, Euglenophyceae 2 y Dinophyceae 1 . Los valores de riqueza específica variaron entre 90 especies (Sitio 1 , deriva, enero) y 4 especies (Sitio 4, peri- 
Tabla 4. Estadísticos descriptivos para los atributos estructurales de deriva y perifiton en los sitios estudiados, 1: arroyo Piedra Blanca; 2: arroyo San Bartolomé; 3: Confluencia, 4: río Chocancharava. Summary statistics for the structural attributes of drift, and periphyton a thet study sites. 1: Piedra Blanca stream, 2: San Bartolome stream, 3: Confluence 4: Chocancharava river

\begin{tabular}{|c|c|c|c|c|c|c|c|c|}
\hline & & & & SITIOS & & & & \\
\hline & & & & & & 3 & & \\
\hline & & perifiton & deriva & perifiton & deriva & deriva & perifiton & deriva \\
\hline Abundancia & Media & 30437 & 7.62 & 48266 & 11.48 & 6.39 & 27040 & 2.56 \\
\hline & Desvio & 29663 & 5.71 & 61712 & 12.68 & 4.83 & 29673 & 2.32 \\
\hline & Max & 109558 & 18.73 & 176721 & 40.58 & 15.36 & 110166 & 8.61 \\
\hline & $\min$ & 3191 & 1.24 & 1192 & 2.21 & 0.87 & 1975 & 0.20 \\
\hline & $\mathrm{CV}$ & 97.46 & 75 & 127.86 & 110.45 & 75.63 & 109.74 & 90.45 \\
\hline Riqueza & Media & 14.54 & 42.15 & 14.92 & 42.54 & 26 & 11.85 & 19.69 \\
\hline & Desvio & 4.59 & 17.50 & 9.99 & 13.32 & 12.23 & 4.54 & 10.38 \\
\hline & $\operatorname{Max}$ & 23 & 90 & 30 & 72 & 54 & 20 & 45 \\
\hline & $\min$ & 9 & 23 & 2 & 28 & 7 & 7 & 4 \\
\hline & $\mathrm{CV}$ & 31.60 & 41.52 & 66.92 & 31.31 & 47.05 & 38.35 & 52.71 \\
\hline Diversidad & Media & 1.70 & 2.33 & 1.72 & 2.39 & 2.00 & 1.60 & 2.01 \\
\hline & Desvio & 0.42 & 0.37 & 0.73 & 0.39 & 0.48 & 0.32 & 0.47 \\
\hline & Max & 2.5 & 2.91 & 2.48 & 2.79 & 2.70 & 2.17 & 2.97 \\
\hline & $\min$ & 1.07 & 1.66 & 0.25 & 1.51 & 1.18 & 1.04 & 1.14 \\
\hline & $\mathrm{CV}$ & 24.87 & 15.87 & 42.70 & 16.12 & 23.94 & 20.08 & 23.44 \\
\hline
\end{tabular}

fiton, marzo). La mayor similitud entre inventarios fue entre el perifiton del arroyo Piedra Blanca y San Bartolomé. Para el análisis de la relación entre ambos ensambles se construyó una matriz de similitud y el mayor porcentaje se observó entre el perifiton de Piedra Blanca (sitio 1) con la deriva del río Chocancharava (sitio 4) (Fig. 2). Se seleccionaron 81 especies que cumplieron con la condición de representatividad en las muestras. De estas 81 especies, 57 (70\%) fueron comunes entre perifiton y deriva, 22 exclusivas de deriva y 2 exclusivas de perifiton.
La mayor densidad de deriva correspondió a especies de Bacillariophycea provenientes del perifiton: Achnanthidium minutissimum, Amphora pediculus, Cocconeis placentula, Navicula perminuta, Nitzschia frustulum y Synedra ulna con una abundancia relativa del $70 \%$ de la clase en el sitio 1. En el sitio 2 lo hicieron: Achnanthidium minutissimum, Cocconeis placentula, Melosira varians, Nitzschia frustulum y Synedra ulna con el $64 \%$ y en los sitios 3 y 4 fueron Achnanthidium minutissimum, Amphora pediculus, Cocconeis placentula, Melosira varians y Synedra ulna las que aportaron

Tabla 5. Análisis de las variaciones espaciales (Lugar) y temporales (Mes) de la abundancia, riqueza S y diversidad de especies H’ en deriva y perifiton en los sitios de estudio. A: ANOVA y K -W: test de Kruskal -Wallis, ${ }^{*} \mathrm{p} \leq 0.05$, ${ }^{* *} \mathrm{p} \leq 0.01, * * *: \mathrm{p} \leq 0.001$. Analyses of the spatial (site) and temporal (month) variations of abundance, richness $S$, and species diversity $H$ ' in dift, and perifiton in the study sites. A: ANOVA and $K$-W: Kruskal-Wallis test, *: $p \leq 0.05, * * p \leq 0.01, * * *: p \leq 0.001$.

\begin{tabular}{|c|c|c|c|c|}
\hline & \multicolumn{2}{|c|}{ Deriva } & \multicolumn{2}{|c|}{ Perifiton } \\
\hline & Lugar & Mes & Lugar & Mes \\
\hline Abundancia & $\mathrm{A}: \mathrm{F}_{(3,48)}=3.94 * *$ & $\mathrm{~A}: \mathrm{F}_{(12,39)}=3.23^{* *}$ & $\mathrm{~A}: \mathrm{F}_{(2,36)}=0.10 \mathrm{~ns}$ & $\operatorname{A~F}_{(12,36)}=5.69^{* * *}$ \\
\hline Riqueza & $\mathrm{A}: \mathrm{F}_{(3,48)}=9.36^{* * *}$ & $\mathrm{~A} \mathrm{~F}_{(12,39)}=3.06^{* *}$ & K-W ns & K-W ns \\
\hline Diversidad & $\mathrm{A}: \mathrm{F}_{(3,48)}=2.99 *$ & $\operatorname{A~F}_{(12,39)}=2.09^{*}$ & K-W ns & $\mathrm{K}-\mathrm{W} *$ \\
\hline
\end{tabular}



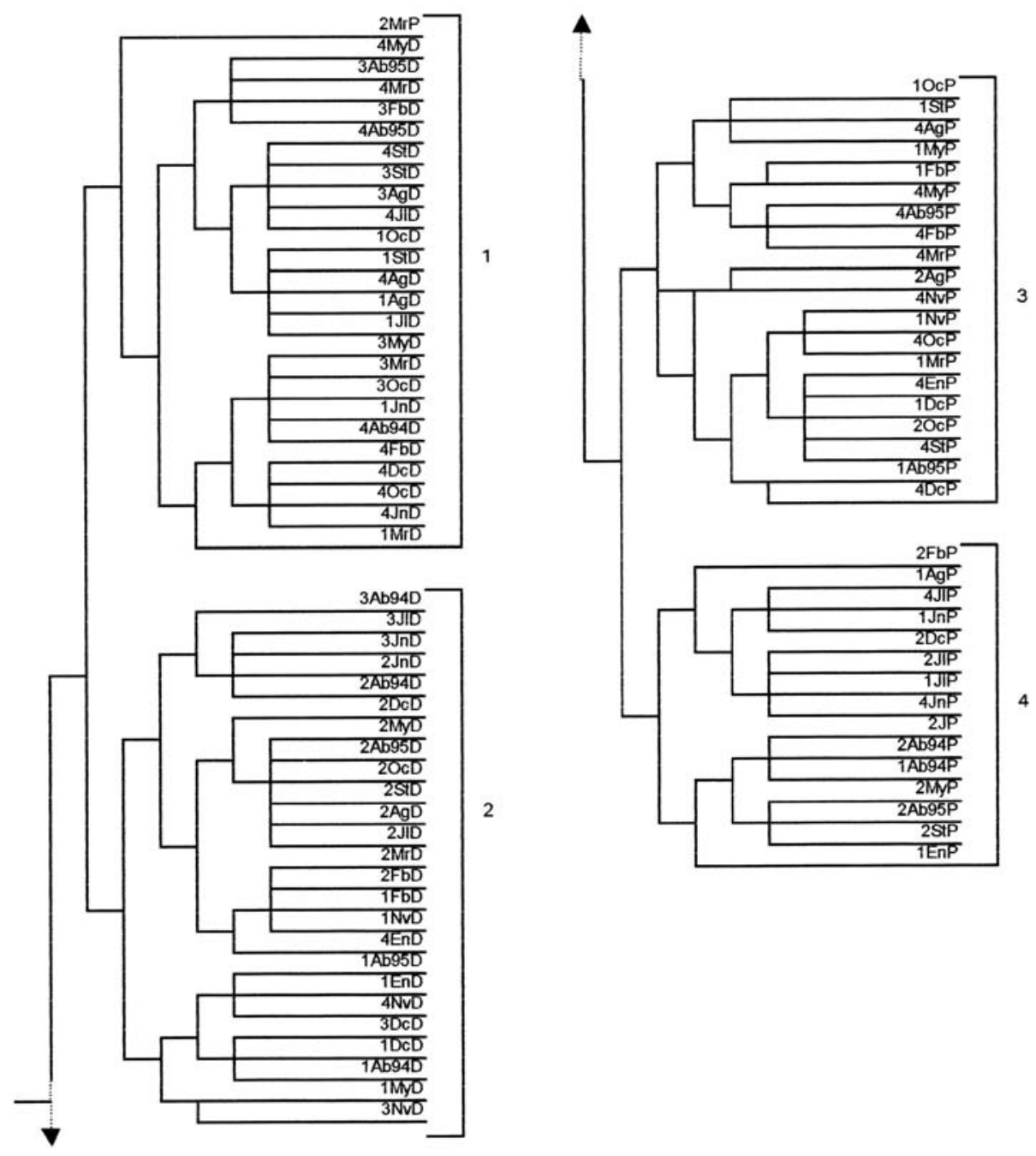

Figura 3. Clasificación de las muestras por TWINSPAN. Los códigos alfa numéricos están formados por lugares: 1,2,3,4; meses y ensambles. (Ej, 2MrP: San Bartolomé, Marzo, Perifiton) GRUPOS: 1- Deriva aguas bajas; 2- Deriva aguas altas; 3:-Perifiton aguas altas; 4-Perifiton aguas bajas. Classification of samples by TWINSPAN. Alpha numeric codes are by places: 1, 2,3,4; months and assemblages (Ex: 2MrP, San Bartolome, march, periphyton). SETS: 1- low water level drift, 2. high water level drift, 3: low water level periphyton, 4. high water level periphyton.

el 83 y $74 \%$ de la densidad total de la clase. La mayor variabilidad espacial correspondió a la abundancia del perifiton (Tabla 4). De la aplicación del ANOVA o del test K-W se evidencian diferencias espaciotemporales con cambios significativos en la abundancia, riqueza y diversidad de la deriva. El perifiton sólo presentó heterogeneidad en la distribución temporal de la abundancia y la diversidad (Tabla 5).
La clasificación de las muestras por TWINSPAN determinó dos grupos: uno que incorporó las muestras de deriva y otro las de perifiton y al interno de cada agrupación se segregaron los conjuntos de aguas altas y aguas bajas (Fig. 3). El ordenamiento de las muestras por DCA en el Eje I (autovalor: 0.67), mostró una correlación significativa $(\mathrm{p}<0.05)$ con la temperatura (r: 0.24) y en el Eje II ( autovalor: 0.30) con la 
composición de especies: riqueza ( $\mathrm{r}: 0.42)$, diversidad H' ( $r$ : 0.36), densidad (r: -0.22$)$ y la velocidad de corriente (r: -0.28), El análisis de correspondencia de las especies indicó un ordenamiento de acuerdo a su pertenencia y abundancia en perifiton o deriva. (Fig. 4).

\section{DISCUSIÓN}

En los sitios estudiados, se observó que el $70 \%$ de las especies fueron comunes a perifiton y deriva. Esta relación varía con los diferentes tipos de arroyos y tramos, ya que se ha encontrado un nivel de participación menor, $50 \%$ (Backhaus, 1969) y 38 \% (Leukart \& Mollenhauer, 1997), evidenciando variaciones en la proporción en la cual el perifiton influye sobre las algas en transporte. Sin embargo, MüllerHaeckel (1973), Swanson \& Bachmann (1976), Stevenson \& Peterson (1991) y Schönborn (1992) concluyeron que las algas suspendidas se originan del perifiton y que existen varios mecanismos que producen el desprendimiento desde el sustrato y posterior ingreso en la deriva. Ellos son: la turbulencia provocada en áreas de rápidos, la formación de burbujas de $\mathrm{O}_{2}$, producto de la gran actividad fotosintética en zonas de baja velocidad de corriente, las características de la morfología celular y la forma de adhesión al sustrato de las diatomeas (Stevenson, 1984; Luttenton \& Rader, 1986; Stevenson \& Peterson, 1989; Biggs et al., 1998).

Las diatomeas, predominantes en el perifiton, constituyen el $80 \%$ de la abundancia relativa de la deriva en los arroyos estudiados, indicando que las especies verdaderamente planctónicas participan con menor densidad.

Con respecto a la composición de especies, los resultados del análisis de similitud, indicaron que la deriva del arroyo de menor orden tuvo menor influencia sobre la composición del perifiton y la deriva del río receptor. En la zona de confluencia, el arroyo tributario de mayor orden predomina en la organización de la composición y atributos de la deriva, probablemente por efecto de la diferencia de los caudales y otros parámetros hidráulicos covariantes con el número de orden (Halminton \& Duthier, 1987, Allan, 1995).

El incremento de la velocidad de corriente actúa como fuerza de estrés provocando la emigración de los organismos, porque la fuerza de arrastre es mayor que la fuerza de adhesión de las diatomeas: firmeza del rafe, presencia de mucílagos y cojín de adhesión (Stevenson, 1983; 1984; Luttenton \& Rader, 1986; Biggs et al., 1998; Bourassa \& Cattaneo, 1998; Saravia et al., 1998; Ghosh \& Gaur, 1998).

El flujo es uno de los factores desencadenantes del desprendimiento de las algas perifíticas (Hamilton \& Duthie, 1987; Power \& Stewart, 1987; Aboal et al., 1996; Abrahamson \& Hakanson, 1998; Passy et al., 1999 ). El efecto del estrés hídrico en la confluencia y el aumento de los caudales, produciría una selección de las especies que colonizan río abajo. Según sus características morfológicas, algunas especies de diatomeas, podrían no encontrar un sustrato apropiado para recolonizar (Müller-Haeckel, 1976; Gustavsson, 1978; Leukart \& Mollenhauer, 1997) como ocurre por ejemplo con Achnanthidium exiguum, Achnanthes inflata, Aulacoseira granulata, Cymbella naviculiformis, Diatoma anceps, Epithemia sorex, especies del arroyo San Bartolomé, que no se encuentran en el río Chocancharava. Otro de los factores del desprendimiento del perifiton es la reproducción celular. Se ha observado en arroyos similares, de baja profundidad, iluminados y con producción primaria perifítica constante, que las células derivantes aumentaron en el horario de mayor actividad fotosintética a consecuencia de la reproducción celular en el perifiton (MüellerHaeckel, 1976; Hamilton \& Duthie, 1987). Con respecto a la variación estacional, el aumento de caudales determina la disminución de su densidad, como se demostró en ríos regulados (Fayolle et al., 1999; Bertrand et al., 2001).

En la deriva el mayor porcentaje de similitud se observó entre el arroyo Piedra Blanca (sitio 1) y el arroyo San Bartolomé (sitio 2), indicando que en la unión se produce un cambio en la composición. En el río receptor se observa un predominio algal procedente del arroyo de mayor jerarquía, mien- 

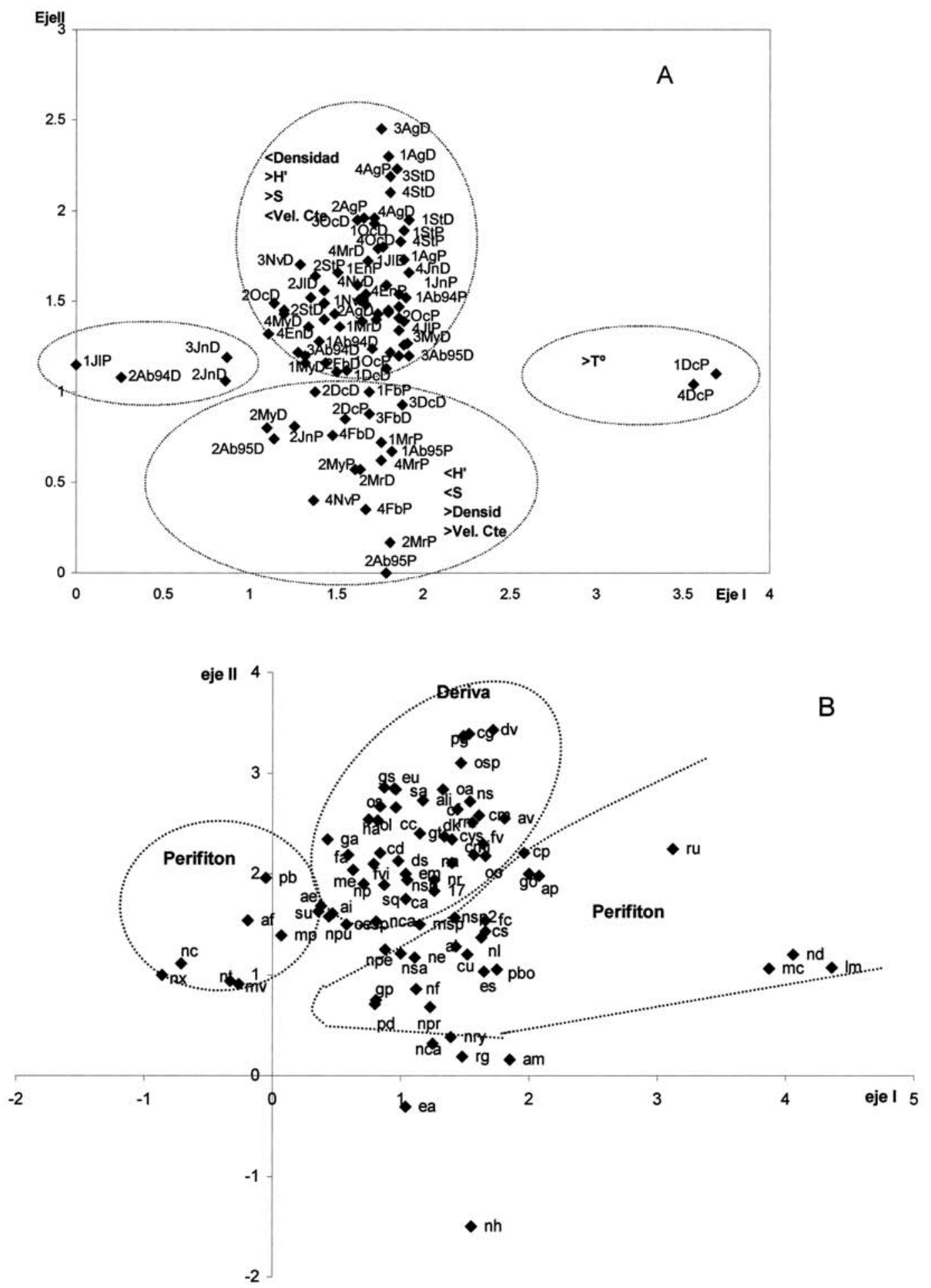

Figura 4. Ordenación mediante Análisis de Correspondencias Detendenciados (DCA) de las muestras (A) y los taxones algales (B) en la confluencia de los arroyos Piedra Blanca y San Bartolomé, 1994-95. Detrented Correspondence Analysis (DCA) ordination of samples (A) and algae taxa (B) at the confluence of Piedra Blanca and San Bartolomé streams, 1994-95. 
tras que la similitud con el arroyo de menor orden es más baja .El arroyo receptor no mantuvo completamente la composición florística de sus tributarios. El encuentro de diferentes conjuntos florísticos que se suceden en la confluencia (Margalef 1994), da origen a una selección de especies organizadas según el orden de los arroyos.

La diversidad y riqueza de especies fue mayor en deriva, porque ésta incluye, además de los organismos del perifiton, otros elementos florísticos componentes del euplancton, del perifiton remoto y de ensambles algales, de diferente origen del analizado como maderas y macrófitas marginales (Sabater, 1990; Corigliano et al., 1994; Sabater et al., 1998; Luque \& Martínez de Fabricius, 2000). Ello explica la baja similitud entre ambos ensambles y su separación en dos grupos diferentes. La densidad de la deriva está relacionada con las especies dominantes del perifiton dado que predominan las mismas poblaciones, entre otras, Achnanthidium minutissimum, Cocconeis placetula, Navicula perminuta.

La dinámica temporal de la densidad y el número de especies estuvo determinada por la estacionalidad térmica e hídrica. Tanto el mayor número de especies, como la mayor densidad en perifiton se observaron en épocas de aguas bajas, mientras que en deriva la mayor riqueza fue en aguas altas. El aumento de los caudales, en verano, época de crecientes, disminuyó la densidad en deriva por efecto de la dilución. Los trabajos sobre el componente algal en transporte en general, no se han dedicado a destacar cuánto de éste es plancton verdadero y cuánto corresponde a organismos del perifiton, pero estudios más detallados al respecto (Müller-Haeckle, 1976; 1978 ) han dado cuenta que en los arroyos el perifiton contribuye con una participación importante en la abundancia de deriva y, en algunos, como en la confluencia de los arroyos Piedra Blanca y San Bartolomé, en forma predominante.

\section{AGRADECIMIENTOS}

Agradecemos al Consejo Nacional de Investigaciones Ciencia y Tecnología (CONI-
CET), al Consejo Nacional de Investigaciones de Córdoba (CONICOR) y a la Secretaría de Ciencia y Técnica de la UNRC (SECYT), los subsidios otorgados para realizar esta investigación. Al laboratorio de Hidrogeología del Departamento de Geología de la U.N.R.C. los análisis químicos de agua.

\section{BIBLIOGRAFÍA}

ABOAL, M., M. A. PUIG \& G. SOLER. 1996. Diatom assemblages in some mediterraneam temporary streams in southeastern Spain. Arch. Hydrobiol., 136(4): 509-527.

ABRAHAMSON, O. \& L. HAKANSON. 1998. Modelling seasonal flow variability of European rivers. Ecol. Model., 114(1): 49-58.

AGUA \& ENERGIA. 1987. Estadística Hidrológica hasta 1983. Tomo I. Fluviometría. Red hidrometeorológica. Buenos Aires. División de Recursos Hídricos.

AMERICAN PUBLIC HEALTH ASSOCIATION (APHA). 1989. Standart Methods for the Examination of Water and Wastewater, $17^{\text {th }}$ ed. APHA, Washington, DC.

ALLAN, D. J. 1995. Stream Ecology. Structure and function of runnning waters. New York: Chapman \& Hall.

ARCHIBALD, R. E. M. 1983. The diatoms of the Sundays and Great at Fish Rivers in the Eastern Cape Province of South Africa. Bibliotheca Diatomologica, 1: 1-362, 34 láms. J. Cramer. Vaduz.

BACKHAUS, D. 1969. Ökologische Untersuchungen an den Aufwuchsalgen der obersten Donau und ihrer Quellflüsse V. Biomassenbestimmungen und Driftmessungen. Arch. Hydrobiol. Suppl., 36 (Donauforschung 4): 1-26.

BERTRAND, C., V. SIAUVE, S. FAYOLLE \& A. CAZAUBON. 2001. Effects of hydrological regime on the drift algae in a regulated Mediterranean river (River Verdon, southeastern France) Regul. Rivers: Res Mgmt., 17: 407-416.

BIGGS , B. J. F., D. G. GORING \& V. I. NIKORA. 1998. Subsidy and stress responses of stream periphyton to gradients in water velocity as a function of community growth form. J. Phycol., 34 (4): 598-607.

BOURASSA, N. \& A. CATTANEO. 1998. Control of periphyton biomass in Laurentian streams (Quebec). J. N. Am. Benthol. Soc., 17(4):420-429. 
BRUNS, D. A., G. W. MINSHALL, C. E. CUSHING, K. W. CUMMINS, J. T. BROCK \& R. L. VANOTE. 1984. Tributaries as modifiers of the river continuum concept :analysis by polar ordination and regression models. Arch. Hydrobiol., 99: 208 - 220.

CORIGLIANO, M. DEL C., A. L. MARTINEZ DE FABRICIUS, E. LUQUE \& N. GARI. 1994. Patrones de distribución de variables físico, químicas y biológicas en el río Chocancharava (Cuarto). Rev.UNRC, 14 (2): 177- 198.

DESIKACHARY, T. V. 1959. Cyanophyta. Ed. Board. New Delhi.

FAYOLLE, S., A. CAZAUBON \& K. COMTE. 1999. Responses and adaptative strategy of epilitic algae communities to different hydrological regimes. Comptes Rendus Acad. Sci. Ser. III Vie-Life Sci., 322 (5): 413-422.

GERMAIN, H. 1981. Flore des Diatomeés. Ed. Boubeé. Paris.

GHOSH, M. \& J. P. GAUR. 1998. Current velocity and the establishment of stream algal periphyton communities. Aquat. Bot., 60(1): 1-10.

GUSTAVSSON, K. 1978. Diel drift of diatoms in an equatorial river. Oikos, 31: 38-40.

HAMILTON, P. B. \& H. C. DUTHIE, 1987. Relationship between algal drift, discharge and stream orden in a boreal forest watershed. Arch. Hydrobiol., 110(2): 275-289.

HILL, M. O. 1979 a. TWINSPAN. A FORTRAN program for arranging multivariate data in an ordered two-way tabla by classification of the individuals and atributes. Ecology and systematic. Cornell University, New York.

HILL, M. O. 1979 b. DECORANA- A FORTRAN program for detrended correspondence analysis and reciprocal averaging. Ecology and systematic. New York: Cornell University.

HYNES, H. B. N. 1970. The Ecology of running waters. Liverpool: University Press

KRAMMER K. \& H. LANGE-BERTALOT. 1986. Bacillariophyceae 2. Naviculaceae. 2/1. In: Süsswasserflora vom Mitteleuropa. G. F. Verlag, Jena.

KRAMMER K. \& H. LANGE-BERTALOT. 1988. Bacillariophyceae 2. Bacillariaceae, Epithemiaceae, Surirellaceae 2/2. In: Süsswasserflora vom Mitteleuropa. Ettl, H. et al. (eds.). G.Fischer Verlag. Jena.

KRAMMER, K. \& H. LANGE-BERTALOT. 1991 a. Bacillariophyceae 3. Teil: Centrales, Fragila- riaceae, Eunotiaceae. 2/3. In: Süsswasserflora vom Mitteleuropa. Ettl, H. et al. (eds.). G.Fischer Verlag. Jena.

KRAMMER, K. \& H. LANGE-BERTALOT. 1991 b. Bacillariophyceae 4. Teil: Achnanthaceae, kritische Ergänzungen zu Navicula (Lineolatae) und Gomphonema Gesamtliteraturverzeichnis 2/4. En: Süsswasserflora vom Mitteleuropa. Ettl, H. et al. (eds.). G.Fischer Verlag. Jena.

LEUKART, P. \& D. MOLLENHAUER. 1997. Studies on algal drift in a small soft-water stream in the Spessart mountains, Germany. Nova Hedwigia, 65 (1-4): 431-442.

LUDWING, J. A. \& J .F. REYNOLDS. 1988. Statistical ecology. A primer on Methods and computing. John Wiley \& Sons. N.Y.

LUQUE, M. E. \& A. L. MARTÍNEZ DE FABRICIUS. 2000. Ficoflora fitoplanctónica y epilítica del río Piedra Blanca (Córdoba, Argentina). Bol. Soc. Argent. Bot., 35 (1-2): 21-32.

LUTTENTON, M. R. \& R. G. RADE, 1986. Effects of disturbance on epiphytic community architecture. J. Phycol., 22: 320-326.

MARGALEF, R. 1994. El río Planetario. Tankay, 1: III-XII.

MARTINEZ DE FABRICIUS, A. L., M. C. FERNANDEZ, N. GARI \& M. DEL C. CORIGLIANO. 1988. Análisis del componente algal en transporte en ríos y arroyos del valle de Calamuchita (Córdoba- Argentina). Rev.UNRC, 8(1): 95-110.

MARTINEZ DE FABRICIUS, A. L. \& M. DEL C. CORIGLIANO. 1989. Composición y distribución de comunidades algales en el Río Ctalamuchita (Córdoba) Rev. UNRC, 9(1): 5-13.

MERRIT, R. W. \& J. B. WALLACE. 1981. FilterFeeding insects. Sci. Am., 244: 132-144.

MÜLLER-HAECKE, A. 1973. Different patterns of synchronization in diurnal and nocturnal drifting algae in the subartic summer. Aquilo Ser. Zool., 14: 19-22.

MÜLLER-HAECKEL, A. 1976. Migrationsperiodik einzelliger Algen in Fliessgewässern. Växtekol. Studier, 10: 1-36.

MÜLLER-HACKEL, A. 1978. The diatomflora of a small stream near Abisko (Swedish Lapland) and its annual periodicity, judged by drift and colonization. Arch. Hydrobiol., 84 (2): 199 - 217.

PASSY, S.I.; Y. D. PAN \& R. L. LOWE. 1999. Ecology of the major periphytic diatom communities from the Mista River Bulgaria. Int. Rev. Hydrobiol., 84 (2): 129-174. 
PATRICK, R. \& C. W. REIMER. 1966. The Diatoms of the United States exclusive of Alaska and Hawai. Vol. 1. Acad. Nat. Sci. Philadelphia. Monog., 13: 1-688.

PATRICK, R. \& C. W. REIMER. 1975. The Diatoms of the United States exclusive of Alaska and Hawai. Vol. 2. Acad. Nat. Sci. Philadelphia. Monog., 13: 1-213.

POWER, M. E. \& A. J. STEWART. 1987. Disturbance and recovery of and algal assemblage following flooding in an Oklahoma stream. Am. Midl. Nat., 117: 333 - 345.

PRESCOTT, G. W., 1962. Algae of the Western Great lake area. Otto Koeltz Science. Germany.

ROUND, F. E. \& L. BUKHTIYAROVA. 1996. Four new genera based on Achnanthes (Achnanthidium) together with a re-definition of Achnanthidium. Diatom Research., 11 (2): 345-361.

ROUND, F. E., R. M. CRAWFORD, \& D. C. MANN. 1990. The diatoms. Biology and morphology of the genera. Cambridge University Press, Cambridge.

SARAVIA, L. A., F. MOMO \& L. D. B. LISSIN. 1998. Modelling periphyton dynamic in running water. Ecol. Model., 114 (1): 35-47.

SABATER, S. 1990. Phytoplankton composition in a medium- sized Mediterranean rivers: The Ter (Spain). Limnetica, 6: 47-56.

SABATER, S., S. V. GREGORY \& J. R. SEDELL. 1998. Community dynamics and metabolism of benthic algae colonizing wood and rock substrata in a forest stream. J. Phycol., 34 : 561-567.

SCHÖNBORN, W., 1992. Fließgewässerbiologie. Stuttgart: G. Fischer, Jena.

STATZNER, B. \& B. HIGLER. 1986. Stream hydraulics as a major determinant of benthic invertebrate zonation patterns. Freshw. Biol., 16: 127-139.

STEVENSON, R. J. 1983. Effects of current and conditions simulating autogenically changing microhabitats on benthic diatom immigration. Ecology, 64(6): 1514-1524.

STEVENSON, R. J. 1984. How currents on different sides of substrates in streams affect mechanisms of benthic algal accumulation. Int. Revue ges. Hydrobiol., 69(2): 241-262.

STEVENSON, R. J. 1986. Importance of variation in algal immigration and growth rates estimated by modeling benthic algal colonization. In: Algal Biofouling L. V. Evans \& K. D. Hoagland (eds.).: 193-210. Elsevier Press, Amsterdam.

STEVENSON, R. J. 1990. Benthic algal community dynamics in a stream during and after a spate. $J$. N. Am. Benthol. Soc., 9(3): 277 - 288.

STEVENSON, R. J. \& C. G. PETERSON. 1989. Variation in Benthic Diatom (Bacillariophyceae) immigration with habitat characteristics and cell morphology. J. Phycol. 25: 120 - 129.

STEVENSON, R. J. \& C. G. PETERSON. 1991. Emigration and immigration can be important determinants of benthic diatom assemblages in streams. Freshw. Biol., 26: 279 - 294.

STRAHLER, A. N. 1981. Geografía Física. Ed. Omega, Barcelona.

SWANSON, C. O. \& R. W. BACHMAN. 1976. A model of algal exports in some Iowa streams. Ecology, 57: 1076 - 1080.

TELL, G. \& V. CONFORTI. 1986. Euglenophyta Pigmentadas de la Argentina. Bibliotheca Phycologica, 75. J. Cramer. Berlin. Stuttgart.

VAN LANDINGHAM, S. L. 1967-1979. Catalogue of the fossil and recent genera and species of diatoms and their synonyms. I-VIII. J. Cramer, Vaduz VÁZQUEZ, J. B., R. A. MIATELLO \& M. E. ROQUE. 1979. IV. Aguas: 168-172 In: Geografia de la Provincia de Córdoba. J.B. Vázquez; R.A. Miatello \& M.E. Roqué (eds.). Boldt, Argentina.

VILLAFAÑE, V. E. \& F. M. H. REID. 1995. Métodos de microscopía para la cuantificación del fitoplancton. En: Manual de Métodos Ficológicos. Alveal, K.; M. E. Ferrario; Oliveira, E. C. \& E. Sar (eds.).: 169-185. Universidad de Concepción. Chile.

WHITTON, B. A. 1991. Aims of monitoring. In: Use of algae for the monitoring rivers. Whitton, B. A; E. Rott \& G. Friedrich (eds.): 5-7. Studia Studentenforderungs-Ges.m.b.H, Austria. 